\title{
Reconnaissance d'émotions faciales, vocales et musicales suivant un traumatisme craniocérébral: état actuel des connaissances
}

Reconocimiento emocional de rostros, voces y música después de un traumatismo de cráneo: estado actual del conocimiento Reconhecimento emocional de faces, voz e música pós-traumatismo cranioencefálico: estado atual do conhecimento Emotional recognition from face, voice and music following a traumatic brain injury: current state of knowledge

\section{Joanie Drapeau $^{1,2}$, Nathalie Gosselin ${ }^{2}, \&$ Michelle McKerral $^{1}$}

\author{
${ }^{1}$ Université de Montréal, Canada. ${ }^{2}$ Laboratoire international de recherche sur le cerveau, la musique et le son (BRAMS), Canada.
}

\section{Résumé}

La reconnaissance émotionnelle apparaît jouer un rôle essentiel dans les relations interpersonnelles. L'habileté à interpréter les émotions dans l'environnement permet en effet aux individus d'anticiper certaines intentions ou situations et d'y répondre adéquatement. Suite à un traumatisme craniocérébral (TCC) apparaissent typiquement des difficultés au regard du fonctionnement psychosocial (p.ex., perturbation des relations intimes, perte d'emploi, effritement du réseau social) qui pourraient être liés à une détérioration dans la reconnaissance des émotions. D'ailleurs, certaines des structures cérébrales les plus importantes pour le traitement émotionnel sont fréquemment atteintes chez les personnes qui ont subi un TCC, telles que les aires frontales, temporales et le système limbique (entre autres, l'amygdale et l'hippocampe). Cet article a pour but de mettre en perspective et d'interroger les connaissances actuelles sur la reconnaissance émotionnelle évoquée par le visage, la voix et la musique des personnes adultes ayant eu un TCC, en comparaison avec une population adulte sans trouble cognitif.

Mots clés : Traumatisme craniocérébral, émotions, visages, voix, musique.

\section{Resumen}

El reconocimiento emocional parece tener un papel clave en las relaciones. Además, la habilidad para interpretar emociones en contexto permite a los individuos anticipar intenciones o situaciones y responder a ellas de modo correcto. Una de las consecuencias típicas del traumatismo encéfalo craneano (TEC) es la dificultad a nivel del funcionamiento psicosocial (por ejemplo, pérdida del trabajo, ruptura de relaciones personales, red social reducida), relacionada con el deterioro del reconocimiento emocional. Más aun, algunas de las estructuras cerebrales más importantes para el procesamiento emocional se ven afectadas en sujetos que han sufrido un TEC, tales como las áreas frontal y temporal y el sistema límbico (amígdala e hipocampo). El objetivo de este trabajo es poner en perspectiva y discutir el conocimiento actual sobre reconocimiento emocional de rostros, voces y música en adultos con TEC, comparado con adultos sin alteraciones cognitivas.

Palabras clave: Traumatismo encéfalo craneano; emociones; rostro; voces; música.

\section{Resumo}

O reconhecimento de emoções parece desempenhar um papel importante nos relacionamentos. Realmente, a habilidade de interpretar emoções no ambiente permite que indivíduos antecipem certas intenções ou situações, e respondam a elas de forma apropriada. Dificuldades de funcionamento psicossocial (por exemplo, perda de trabalho, ruptura de relacionamentos íntimos, reduzida rede social) tipicamente aparece após um traumatismo cranioencefálico (TCE), o que pode estar relacionado com a deterioração do reconhecimento emocional. Além disso, estruturas cerebrais importantes para o processamento emocional é frequentemente afetada em indivíduos após um TCE, como áreas frontais e temporais, e o sistema límbico (como a amígdala e o hipocampo). Esse artigo tem como objetivo colocar em perspectiva e questão o conhecimento atual sobre o reconhecimento emocional de faces, vozes e música em adultos com TCE, quando comparados a adultos sem prejuízo cognitivo.

Palavras-chave: Traumatismo cranioencefálico; emoções; faces; voz; música.

Artícle reçu: 14/10/2011; Artícle revisé: 28/12/2011; Artícle accepté: 29/12/2011.

Joanie Drapeau, Centre de recherche interdisciplinaire en réadaptation-Centre de Réadaptation Lucie-Bruneau et Département de psychologie, Université de Montréal, Montréal, Québec, Canada; Laboratoire international de recherche sur le cerveau, la musique et le son (BRAMS), Montréal, Québec, Canada.

Nathalie Gosselin, Laboratoire international de recherche sur le cerveau, la musique et le son (BRAMS), Montréal, Québec, Canada.

Michelle McKerral, Centre de recherche interdisciplinaire en réadaptation-Centre de Réadaptation Lucie-Bruneau et Département de psychologie, Université de Montréal, Montréal, Québec, Canada.

Adresse de correspondance: Professeure Michelle McKerral, Centre de recherche interdisciplinaire en réadaptation-Centre de Réadaptation LucieBruneau, 2275 avenue Laurier est, Montréal, Québec, Canada H2N 2N8. Tél.: 1-514-343-2336; Fax: 1-514-343-5787.

E-mail: michelle.mcKerral@ umontreal.ca

DOI: $10.5579 / \mathrm{rnl} .2011 .0075$ 
Emotional recognition appears to play a key role in relationships. Indeed, the ability to interpret emotions in the environment allows individuals to anticipate certain intentions or situations, and to respond to them in an appropriate fashion. Difficulties in psychosocial functioning (e.g., job loss, disruption of intimate relationships, reduced social network) typically appear following a traumatic brain injury (TBI), which may be related to deterioration in emotional recognition. Moreover, some of the most important brain structures for emotional processing are frequently affected in individuals having sustained a TBI, such as frontal and temporal areas, and the limbic system (such as the amygdala and hippocampus). This article aims to put into perspective and to discuss the current knowledge on emotional recognition from faces, voices and music in adults with TBI, as compared to adults without cognitive impairment.

Key words: Traumatic brain injury, emotions, face, voice, music.

Selon les données d'études épidémiologiques canadiennes récentes (Cassidy et al., 2004; Zygun et al., 2005), l'incidence annuelle du traumatisme craniocérébral (TCC) est estimée à 600 personnes touchées pour 100000 habitants dans le cas d'un TCC de sévérité légère et à 11,4 personnes sur 100000 dans le cas d'un TCC de sévérité grave. Le TCC constitue par ailleurs une des causes principales de décès et d'incapacités chez les individus de moins de 35 ans au Québec (Regroupement des associations de personnes traumatisées craniocérébrales du Québec, 2005).

Les causes du TCC sont variables chez les adultes: accidents de la route (45\%), chutes (30\%), accidents de travail (10\%), sports et loisirs (10\%) et agressions physiques (5\%) (Institut canadien d'information sur la santé [ICIS], 2007). De tels accidents peuvent entraîner d'importantes conséquences, permanentes et irréversibles dans la plupart des cas, sur les plans physique (p.ex., fatigue, trouble du sommeil, maux de tête, douleurs corporelles), cognitif (attention/concentration, mémoire à court terme, vitesse de traitement, fonctions exécutives), affectif (instabilité émotionnelle, dépression, anxiété, colère/irritabilité) et/ou comportemental (impulsivité, désinhibition, comportement sexuel inapproprié, manque d'initiative, changements dans la personnalité) (Hibbard et al., 1998).

Les changements cognitifs sont souvent les plus saillants à la suite d'un TCC, quelque soit la sévérité, et il s'agit là des problèmes les plus fréquemment cités par les patients et leurs aidants dans le cas d'un TCC grave (McCullagh \& Feinstein, 2005). Les études s'intéressant aux conséquences du TCC sur la cognition se sont particulièrement centrées sur ses effets sur l'attention, l'apprentissage, la mémoire, le langage et les fonctions exécutives (p.ex., McCullagh \& Feinstein, 2005, pour une revue). La reconnaissance émotionnelle est pour sa part une fonction cognitive encore peu étudiée chez les personnes ayant subi un TCC. Le domaine des émotions a été davantage exploré selon une perspective psychologique et psychiatrique, les écrits ayant investigué plus particulièrement les troubles affectifs post-accidents (p.ex., dépression, anxiété) (Robinson \& Jorge, 2005; Warden \& Labbate, 2005).

Par ailleurs, la majorité des personnes ayant subi un TCC démontre typiquement des difficultés au regard du fonctionnement psychosocial (p.ex., perte d'emploi, perturbation des relations intimes, effritement du réseau social) (Dahlberg et al., 2006; De Guise et al., 2008; Gosling \& Oddy, 1999; Hammond, Hart, Bushnik, Corrigan \& Sasser, 2004; Kersel Marsh, Havill \& Sleigh, 2001; Morton \& Wehman, 1995). Bien que les causes de ces difficultés psychosociales suivant un TCC demeurent équivoques, certains auteurs ont soulevé qu'elles pourraient être liées à une détérioration de l'habileté à reconnaître les émotions (p.ex., Hornak, Rolls \& Wade, 1996; Knox et Douglas, 2009; Kubu et al., 1993; Milders, Ietswaart, Crawford \& Currie, 2008). En effet, la reconnaissance émotionnelle joue un rôle essentiel dans les relations interpersonnelles. La capacité à interpréter les émotions dans l'environnement permet aux individus d'anticiper certaines intentions/situations (positives ou négatives) et d'y répondre adéquatement (Bibby \& McDonald, 2005). Il est donc possible qu'une reconnaissance déficitaire des émotions puisse en partie affecter le fonctionnement social des personnes ayant subi un TCC.

Par le biais d'une recension des écrits, cet article vise à mettre en perspective et à interroger les connaissances actuelles au regard de la reconnaissance émotionnelle des personnes adultes ayant subi un TCC, en comparaison avec une population adulte sans trouble cognitif. Plus précisément, la reconnaissance d'émotions évoquée par les expressions faciales, les expressions vocales et la musique sera abordée. Le visage et la voix sont les stimuli non-verbaux les plus communs et les plus puissants pour communiquer les émotions (p.ex., joie, tristesse, peur) (Johnstone \& Scherer, 2000; Keltner \& Ekman, 2000). Pour sa part, la musique, exploitée dans le domaine des émotions (p.ex., Juslin, 2001), constitue également un médium de communication considérable et est d'ailleurs utilisée comme intervention en clinique pour répondre aux besoins de prise en charge comportementale, cognitive et/ou émotionnelle des personnes présentant ou non des atteintes cérébrales (p.ex., Baker \& Tamplin, 2006; Bunt, 2001).

\section{Corrélats cérébraux du TCC et de la reconnaissance d'émotions}

Le TCC est un événement mécanique, provoqué par des forces physiques externes et entrainant des lésions au niveau du crâne et de son contenu (Cohadon, 1992). Les atteintes cérébrales se produisent en raison d'un effet de contact ou d'impact (i.e., coup et contre-coup), d'un effet d'inertie (i.e., accélération et/ou décélération), ou d'une combinaison des deux (Povlishock \& Katz, 2005). L'effet de contact est observé lorsque la tête heurte ou est heurtée par un objet. Les lésions sont d'abord locales, à la surface d'impact, produisant des plaies, des contusions à des degrés divers ou une fracture de la voûte crânienne. L'effet d'inertie est observé lorsque la tête est mise en mouvement (accélération) ou est arrêtée dans son mouvement (décélération) (Cohadon, 1992). Les forces d'inertie étirent et cisaillent la matière blanche dans certaines aires cérébrales (i.e., la partie supérieure du tronc cérébral, les régions para-sagittales de la matière blanche du cerveau, le corps calleux et les jonctions de la matière grise et de la matière blanche du cortex cérébral), ayant pour résultat des lésions axonales diffuses (ou 
plus exactement, multifocales; Meythaler, Peduzzi, Eleftheriou \& Novack, 2001).

Par ailleurs, plusieurs structures cérébrales sont touchées lors de ces forces mécaniques. De nombreuses études quantitatives de résonance magnétique ont noté que les régions cérébrales les plus fréquemment et les plus sévèrement atteintes à la suite d'un TCC (particulièrement de sévérité modérée ou grave) sont les aires frontales et temporales (p.ex., Anderson, Bigler \& Blatter, 1995; Bigler, 1999; Bigler, Anderson \& Blatter, 2002; Gale et al., 1995; Umile, Sandel, Alavi, Terry \& Plotkin, 2002), le système ventriculaire (p.ex., Bigler et al., 1984; Bigler, Kurth, Blatter \& Abildskov, 1992; Blatter et al., 1997; Cullum \& Bigler, 1986; Thorley, Wertsch \& Klingbeil, 2001) et le corps calleux (p.ex., Huisman et al., 2004; Johnson, Pinkston, Bigler \& Blatter, 1996; McGowan et al., 2000). Certaines régions du système limbique (i.e., amygdale, hippocampe, fornix) peuvent également être atteintes de manière importante lors d'un TCC (p.ex., Arciniegas et al., 2001; Bigler, 1999; Bigler et al., 1996; Gale, Burr, Bigler \& Blatter, 1993; Jantzen, Anderson, Steinberg \& Kelso, 2004; Tate \& Bigler, 2000). De plus, les études récentes démontrent que même dans le cas de TCC de sévérité légère, des changements structuraux et altérations neurochimiques surviennent dans ces mêmes aires cérébrales, ainsi que dans l'intégrité de la matière blanche (Beauchamp et al., 2011; Henry et al., 2010; Mayer et al., 2011; Yeo et al., 2011).

La capacité à traiter les émotions peut être particulièrement vulnérable chez les personnes ayant subi un TCC, puisque certaines des aires cérébrales touchées sont impliquées dans la reconnaissance émotionnelle. En effet, plusieurs études de lésions et de neuroimagerie, menées chez des animaux et des humains, ayant fournit un portrait d'ensemble de l'architecture neuronal du traitement émotionnel ont montré de manière générale que les divers sous-systèmes interactifs impliqués dans la reconnaissance d'émotions sont principalement situés dans les régions frontales et temporales du cerveau. Ces systèmes incluent le système limbique (p.ex., amygdale), le gyrus cingulaire antérieur, le cortex insulaire, le cortex pariétal, le cortex somatosensoriel, et le secteur ventral du lobe frontal (p.ex., pour une revue, Adolphs, 2002; Adolphs \& Damasio, 2000; Phillips, Drevets, Rauch \& Lane, 2003).

La reconnaissance émotionnelle implique également de manière spécifique différentes structures cérébrales, selon le médium de communication utilisé pour évoquer les émotions (i.e., le visage, la voix et la musique). Au regard de la reconnaissance d'émotions évoquées par le visage, diverses études ont suggéré que le cortex somatosensoriel droit (Adolphs, Damasio, Tranel, Cooper \& Damasio, 2000; Adolphs, Damasio, Tranel \& Damasio, 1996), le cortex visuel temporal (Critchley et al., 2006; Haxby, Hoffman \& Gobbini, 2002; Streit et al., 1999; Weddell, 1994), le cortex cingulaire antérieur (Hornack et al., 2004; Streit et al., 1999), le gyrus temporal médian (Pourtois, de Gelder, Bol \& Crommelink, 2005), les cortex préfrontal médian et orbitofrontal (Blair, Morris, Frith, Perrett, \& Dolan, 1999; Damasio, 1994; Hornack, Rolls \& Wade, 1996; Hornack et al., 2003; Phillips, Drevets, Rauch \& Lane, 2003) et le lobe temporal antéromédian (Gosselin, Peretz \& Samson, sous presse) sont impliqués. Le système neuronal cérébral s'avère être également spécialisé pour la reconnaissance de certaines catégories d'émotions faciales. En effet, l'amygdale, une structure cérébrale du système limbique, possède un rôle central dans la réponse face au danger, et en particulier dans la réponse de peur (Adolphs \& Tranel, 1995). Il a notamment été démontré qu'une lésion bilatérale des noyaux amygdaliens peut être reliée à un déficit de la reconnaissance de la peur évoquée par le visage (p.ex., Adolphs, Tranel, Damasio, \& Damasio, 1994). Plus récemment, Gosselin, Peretz et Samson (sous presse) ont démontré une reconnaissance déficitaire des visages exprimant la peur suite à une résection du lobe temporal antéromédian (incluant l'amygdale). Par ailleurs, une spécialisation fonctionnelle de chacun des deux hémisphères cérébraux dans le traitement d'émotions est observée, où deux hypothèses sont souvent discutées: l'hypothèse de l'hémisphère droit indique que celui-ci se spécialise dans le traitement de toutes les émotions évoquées par des stimuli non-verbaux (Bowers, Bauer \& Heilman, 1993), alors que l'hypothèse de valence soutient que l'hémisphère droit se spécialise dans le traitement d'émotions négatives alors que l'hémisphère gauche s'implique davantage dans le traitement d'émotions positives (p.ex., Davidson, 1992). La contribution hémisphérique de la reconnaissance d'émotions faciales n'est cependant pas encore claire aujourd'hui. L'hypothèse de valence est soutenue par Adolphs, Damasio, Tranel et Damasio (1996), qui ont démontré que les personnes cérébrolésées droites présentent un déficit dans la reconnaissance d'émotions faciales négatives (peur, tristesse) et une préservation de la reconnaissance d'émotions faciales positives (joie). Néanmoins, les résultats de Best, Womer et Queen (1994) supportent davantage l'hypothèse de l'hémisphère droit, où les auteurs ont plutôt montré une supériorité du champ visuel gauche (i.e., hémisphère droit) pour les expressions négatives ainsi que pour les expressions positives exprimées par le visage.

Concernant la reconnaissance émotionnelle évoquée par la voix, certaines études soutiennent l'implication de l'opercule frontopariétal droit, du pôle frontal bilatéral, du sillon temporal supérieur, du cortex temporal, de l'insula, du gyrus frontal inférieur (Adolphs, 2002; Grandjean et al., 2005; Pell, 2006; Schirmer \& Kotz, 2006), du gyrus temporal médian (Pourtois, de Gelder, Bol \& Crommelink, 2005), du cortex préfrontal ventromédian, du cortex orbitofrontal et du cortex cingulaire antérieur (George et al., 1996; Morris, Scot, \& Dolan, 1999; Peretz, 2009, pour une revue; Phillips, Drevets, Rauch \& Lane, 2003). Le système neuronal s'avère être également spécialisé pour la reconnaissance de certaines catégories d'émotions vocales. Des études ont démontré que la reconnaissance de la peur évoquée par la voix est altérée suite à des lésions de l'amygdale (Scott et al., 1997; Sprengelmeyer et al., 1999) et des données de neuroimagerie montrent également l'activation de l'amygdale pour les expressions de peur évoquées par des stimuli vocaux (Dolan, Morris \& de Gelder, 2001; Fecteau, Belin, Joanette \& Armony, 2007; Morris et al., 1996; Phillips et al., 1998). Par ailleurs, les émotions vocales sont associées typiquement à l'hémisphère droit (hypothèse de l'hémisphère droit), démontré par l'étude de patients cérébrolésés droits (p.ex., Schirmer \& Kotz, 2006) et par le biais de techniques d'imagerie cérébrale (p.ex., imagerie par résonance 
magnétique [IRM], Mitchell, Elliott, Barry, Cruttenden et Woodruff, 2003).

Au regard de la reconnaissance d'émotions évoquées par la musique, certains auteurs ont montré l'implication du cortex orbitofrontal (Blood \& Zatorre, 2001; Blood, Zatorre, Bermudez \& Evans, 1999; Menon \& Levitin, 2005), du cortex temporal supérieur et du cortex cingulaire antérieur (Blood \& Zatorre, 2001; Blood, Zatorre, Bermudez \& Evans, 1999; Mitterschiffthaler, Fu, Dalton, Andrew \& Williams, 2007; Green et al., 2008). De plus, certains auteurs ont trouvé, en utilisant la tomographie par émission de positons (TEP), que les régions paralimbiques et néocorticales étaient impliquées dans la reconnaissance de musiques plaisantes (Blood, Zatorre, Bermudezt \& Evans, 1999). Par ailleurs, le rôle de l'amygdale a été mis en évidence dans la reconnaissance de la peur évoquée par la musique. Gosselin et al. (2005, 2007, sous presse) ont démontré une performance significativement diminuée pour la reconnaissance de la peur évoquée par la musique chez des patients présentant des lésions de l'amygdale. Deux études de neuroimagerie ont également observé une activité augmentée de l'amygdale lors du visionnement de films présentant une trame sonores évoquant la peur (Baumgartner, Lutz, Schmidt \& Jancke, 2006; Eldar, Ganor, Admon, Bleich \& Hendler, 2007). Tout comme pour le visage, la contribution hémisphérique de la reconnaissance d'émotions musicales est équivoque. Plusieurs études ayant mesuré l'activité électrique de cerveau (EEG) ont démontré une plus grande activité EEG gauche lors de l'écoute d'extraits musicaux plaisants et une plus grande activité EEG droite pour les musiques désagréables (Altenmuller, Schurmann, Lim \& Parlitz, 2002; Flores-Gutierrez et al., 2007; Schmidt \& Trainor, 2001; Tsang, Trainor, Santesso, Tasker \& Schmidt, 2001). De manière similaire, Gagnon et Peretz (2000) ont démontré, à partir d'un modèle d'asymétries auditives chez des auditeurs normaux, un effet de supériorité de l'oreille gauche (prédominance de l'hémisphère droit) à l'écoute de musiques désagréables, et un léger avantage de l'oreille droite (prédominance de l'hémisphère gauche) à l'écoute de musiques plaisantes (hypothèse de valence). Toutefois, d'autres études observent d'avantage un avantage global de l'hémisphère droit pour la musique positive ou négative (Bryden, Ley \& Sugarman, 1982), ainsi que pour la musique plaisante ou consonante (hypothèse de l'hémisphère droit) (Blood, Zatorre, Bermudez, \& Evans, 1999).

Bien que la reconnaissance d'émotions évoquées par ces trois médiums de communication implique des aires cérébrales similaires, il n'est pas clair si les voies neuronales pour la reconnaissance émotionnelle faciale, vocale et musicale soient partagées (p.ex., Peretz, 2001, 2009, pour une revue). Ainsi, il est possible qu'une personne cérébrolésée ayant un déficit de la reconnaissance émotionnelle évoquée par l'un de ces médiums présente également (ou ne présente pas) un déficit de la reconnaissance émotionnelle évoquée par un autre médium.

\section{Reconnaissance d'émotions faciales et vocales suite à un TCC}

Parmi les quelques études ayant examiné le traitement émotionnel des expressions faciales et de la voix chez les personnes ayant subi TCC, un certain nombre d'auteurs ont conclu à une atteinte. Toutefois, la majorité de ces études ont évalué des personnes qui ont subi leur accident depuis plusieurs années (Croker \& McDonald, 2005;
Dimoska, McDonald, Pell, Tate \& James, 2010; Jackson \& Moffat, 1987; Hopkins, Dywan et Segalowitz, 2002; Knox \& Douglas, 2009; McDonald \& Saunders, 2005; Spell \& Frank, 2000), ce qui rend difficile l'interprétation des résultats au regard de l'implication d'autres variables (p.ex., réorganisation fonctionnelle du cerveau, changements dans l'environnement social) et qui ne permet pas de dresser un portrait de la capacité des personnes TCC à reconnaître les émotions dans les premiers mois suivant le TCC, en vue d'offrir une intervention spécifique.

Seules trois études ont évalué des personnes ayant récemment subi un TCC (Green, Turner \& Thompson, 2004; Ietswaart, Milders, Crawford, Currie et Scott, 2008; Milders, Ietswaart, Crawford et Currie, 2008). Green, Turner et Thompson (2004) ont évalué des personnes ayant subi un TCC grave en moyenne 2,6 mois auparavant, comparativement à des personnes adultes sans trouble cognitif, en utilisant une tâche de reconnaissance d'émotions (i.e., joie, tristesse, colère, peur et neutralité) évoquées par le visage. La performance des personnes TCC était significativement inférieure comparativement aux personnes adultes contrôles, ce qui supporte l'existence d'un déficit dans la reconnaissance d'émotions évoquées par le visage dans les premiers mois post-accident. Milders, Ietswaart, Crawford et Currie (2008) et Ietswaart, Milders, Crawford, Currie et Scott (2008) ont également réalisés une étude permettant de déterminer la capacité des personnes à reconnaître les émotions quelques mois post-accident, mais également l'évolution de leurs performances à long terme. Ainsi, les auteurs ont évalué en deux temps (i.e., une première fois, environ 2,1 mois suivant le TCC; et une deuxième fois, environ 1 an post-accident) des personnes adultes ayant subi un TCC de sévérité légère à grave et des adultes sans trouble cognitif sur la base d'une tâche de reconnaissance d'émotions (peur, dégoût, colère, joie, tristesse et surprise) évoquées par le visage et une tâche de reconnaissance d'émotions (joie, tristesse, colère, peur, neutralité) évoquées par l'intonation de la voix, où les participants devaient indiquer quelle émotion correspond le mieux au stimulus. À la première et à la deuxième évaluation, les personnes ayant subi un TCC ont significativement moins bien performé que les personnes adultes sans trouble cognitif pour chacune des tâches, ce qui supporte la conclusion d'une reconnaissance déficitaire d'émotions évoquées par le visage et la voix chez les personnes ayant récemment subi un TCC, ainsi que suivant le TCC à plus long terme (un an post-trauma).

Par ailleurs, la majorité des études portant sur la reconnaissance émotionnelle évoquée par le visage et la voix suivant un TCC (Dimoska, McDonald, Pell, Tate \& James, 2010; Hopkins, Dywan \& Segalowitz, 2002; Ietswaart, Milders, Crawford, Currie \& Scott, 2008; Milders, Ietswaart, Crawford \& Currie, 2008; Spell \& Frank, 2000) ont inclus des participants dont la sévérité du TCC n'était pas précisée ou différenciée dans les analyses statistiques, alors qu'il peut y avoir des écarts importants dans la capacité à traiter les émotions selon que la personne ait subi un TCC léger, modéré ou grave. Les études ayant distingué clairement les niveaux d'atteintes des participants ont porté seulement sur des personnes ayant subi un TCC grave (Croker \& McDonald, 2005; Green, Turner \& Thompson, 2004; Knox \& Douglas, 2009; Jackson \& Moffat, 1987; McDonald \& Flanagan, 2004; McDonald \& Saunders, 2005; Milders, Ietswaart, Crawford \& 
Currie, 2008), empêchant toute conclusion concernant la performance des personnes ayant subi un TCC léger ou modéré.

Également, il est possible que la présence d'un trouble de reconnaissance émotionnelle post-TCC soit restreinte à seulement certaines catégories d'émotions. En effet, certains auteurs ont indiqué que la reconnaissance d'émotions négatives évoquées par le visage et la voix est diminuée chez les personnes ayant subi un TCC (Hopkins, Dywan \& Segalowitz, 2002; Ietswaart, Milders, Crawford, Currie \& Scott, 2008; Jackson \& Moffat, 1987; Spell \& Frank, 2000). Plus spécifiquement, la peur, la tristesse et/ou la colère évoquée par le visage et la voix se sont avérées significativement moins bien reconnues pour les personnes ayant subi un TCC comparativement aux personnes adultes sans trouble cognitif. La possibilité d'une reconnaissance émotionnelle restreinte à certaines catégories d'émotions est également appuyée par le type d'atteinte cérébrale présente suivant un TCC. En effet, certaines régions cérébrales pouvant être touchées lors du TCC s'avèrent être associées de manière spécifique à diverses catégories d'émotions. Notamment, les noyaux amygdaliens sont reliés à la reconnaissance de la peur évoquée par le visage (Adolphs, Tranel, Damasio, \& Damasio, 1994 ; Gosselin, Peretz \& Samson, sous presse), ainsi que par la voix (Morris, Scott, \& Dolan, 1999; Scott et al., 1997). Ainsi, un déficit de la reconnaissance d'émotions post-TCC pourrait se limiter seulement à la reconnaissance de certaines émotions négatives (p.ex., peur).

En outre, il est possible que les difficultés de reconnaissance d'émotions évoquées par le visage et la voix préalablement observées chez les personnes ayant subi un TCC soient en partie conséquentes d'un trouble à percevoir les caractéristiques du visage et de la voix qui sont cruciales pour la reconnaissance des expressions faciales et des expressions vocales. Plusieurs études n'ont pas utilisé de tâche perceptuelle (p.ex., Knox \& Douglas, 2009; McDonald \& Flanagan, 2004; McDonald \& Saunders, 2005; Milders, Ietswaart, Crawford \& Currie, 2008; Spell \& Frank, 2000), alors qu'il apparaît essentiel de considérer, en parallèle à la reconnaissance de catégories d'émotions, la capacité à discriminer le visage et la voix, afin d'éliminer la possibilité d'un trouble de perception sous-jacent aux déficits de la reconnaissance émotionnelle. Les quelques études ayant utilisé une tâche de perception (Croker \& McDonald, 2005; Hopkins, Dywan \& Segalowitz, 2002; Ietswaart, Milders, Crawford, Currie \& Scott, 2008) ont toutefois montré une atteinte dans la capacité à discriminer les émotions chez les personnes ayant subi un TCC, sur la base de tâches de discrimination $\mathrm{du}$ visage (i.e., indiquer quel visage est le même que celui présenté comme modèle) et de tâches de discrimination de l'intonation de la voix (i.e., indiquer si deux phrases expriment une émotion similaire ou différente). Bien que les auteurs aient justifié que la majorité des participants performaient tout de même au dessus de la normale selon leur âge et leur niveau de scolarité, la conclusion d'une reconnaissance déficitaire d'émotions évoquées par le visage sous-jacente à un trouble perceptuel est équivoque.

Il est aussi à noter que la majorité des études ayant exploré la reconnaissance d'émotions évoquées par le visage auprès d'une population ayant subi un TCC ont utilisé des stimuli visuels statiques (p.ex., Croker \& McDonald, 2005; Green, Turner \& Thompson, 2004; Hopkins, Dywan \& Segalowitz, 2002; Ietswaart, Milders, Crawford, Currie \& Scott, 2008; Jackson \& Moffat, 1987; Knox \& Douglas, 2009; McDonald \& Flanagan, 2004; McDonald \& Saunders, 2005; Milders, Ietswaart, Crawford \& Currie, 2008; Spell \& Frank, 2000), ce qui exclut totalement la dimension temporelle et ne rend possiblement pas compte des capacités réelles des participants. En effet, la reconnaissance d'émotions évoquées par des expressions faciales dynamiques pourrait être plus adéquate, puisqu'elle s'avère être plus représentative de la réalité (i.e., plus écologique). Par ailleurs, il a été démontré que la reconnaissance d'émotions est significativement améliorée lorsqu'évoquée par des visages dynamiques chez des participants adultes normaux (p.ex., Ambadar, Schooler \& Cohn, 2005).

Seules deux études ont utilisé une tâche de reconnaissance d'émotions évoquées par des expressions faciales dynamiques auprès de personnes ayant subi un TCC (Knox \& Douglas, 2009; McDonald \& Saunders, 2005). McDonald et Saunders (2005) ont évalué des personnes ayant subi un TCC grave et des personnes adultes sans trouble cognitif sur la base de tâches de reconnaissance d'émotions (i.e., joie, surprise, tristesse, colère, peur, dégoût et neutralité) évoquées par des expressions faciales dynamiques (i.e., vidéovignettes d'acteurs qui interagissent dans des situations de tous les jours) et statiques. Les personnes ayant subi un TCC ont performé de manière similaire aux personnes adultes contrôles dans la reconnaissance d'émotions évoquées par les stimuli visuels (dynamiques et statiques). Cependant, les auteurs ont noté que la reconnaissance d'expressions faciales statiques a été problématique pour une proportion significative de personnes ayant subi un TCC. Knox et Douglas (2009) ont également évalué des personnes ayant subi un TCC grave par le biais de tâches de reconnaissance d'émotions évoquées par des expressions faciales statiques et dynamiques (la même tâche expérimentale qu'utilisée par McDonald \& Saunders, 2005), où les participants avaient à indiquer l'émotion (i.e., joie, tristesse, colère, peur, surprise et dégoût) qui correspond le mieux au stimulus. Les personnes ayant subi un TCC ont performé de manière inférieure comparativement aux personnes adultes sans trouble cognitif, où les visages dynamiques ont été plus difficiles à reconnaitre que les visages statiques. Ainsi, bien que ces deux études susmentionnées aient évalué des personnes ayant subi un TCC grave (donc présentant le même niveau d'atteinte) et par le biais de la même tâche expérimentale, les auteurs montrent des résultats contradictoires. La divergence de ces résultats pourrait s'expliquer par la nature de la tâche. En effet, celle-ci n'a peut-être pas été bien contrôlée pour examiner spécifiquement les expressions faciales dynamiques. Les auteurs ont spécifié aux personnes de se concentrer uniquement sur le visage des acteurs, mais d'autres indices semblaient être disponibles dans les scènes exposés aux participants (p.ex., gestes, posture).

Par ailleurs, la plupart des études ayant évalué la reconnaissance d'émotions évoquées par la voix ont utilisé une tâche vocale avec un contenu sémantique affectif neutre (i.e., le contenu sémantique est neutre au regard de l'intonation affective de la voix) ou une tâche vocale avec un contenu sémantique affectif incongru (i.e., la phrase ne fait 
aucun sens), qui ont pu paraître complexes pour une population ayant subi un TCC (Dimoska, McDonald, Pell, Tate \& James, 2010; Ietswaart, Milders, Crawford, Currie \& Scott, 2008; McDonald \& Saunders, 2005; Milders, Ietswaart, Crawford \& Currie; 2008; Spell \& Frank, 2000). Ces deux types de tâches impliquent des phrases au contenu sémantique neutre (p.ex., «I'll be back later », Spell \& Frank, 2000) ou des phrases sémantiquement incongrues (p.ex., «Someone migged the pazing », Dimoska, McDonald, Pell, Tate \& James, 2010) et donc, non-concordantes avec l'intonation de la voix. Il s'agit là de stratégies non-familières, qui sollicitent possiblement la capacité à inhiber la tendance naturelle à traiter à la fois l'intonation de la voix et le contenu verbal sémantique d'une phrase. De plus, il a été démontré que les ressources attentionnelles permettant l'inhibition sont souvent altérées chez les personnes ayant subi un TCC (p.ex., Gronwall, 1987; Kewman, Yanus \& Kirsh, 1988; SchmitterEdgecombe \& Kibby, 1998; Van Zomeren \& Brouwer, 1994). Ainsi, les tâches vocales utilisées dans les études antérieures ont pu être trop complexes, puisque les personnes ayant subi un TCC ont de faibles ressources attentionnelles, par ailleurs nécessaires à l'inhibition de la tendance spontanée à traiter le contenu sémantique d'une phrase en concordance avec l'intonation de la voix. Une tâche vocale qui n'interfère pas avec le contenu verbal sémantique apparait donc essentielle à privilégier (p.ex., cris, pleurs, rires, etc.).

Reconnaissance d'émotions évoquées par la musique après un TCC

À notre connaissance, aucune étude ne s'est intéressée au traitement émotionnel via la musique suivant un TCC. Pourtant, la musique (p.ex., écoute, composition, chant) est utilisée comme méthode d'intervention auprès de personnes présentant des problématiques cognitives, comportementales et émotionnelles suite à une atteinte cérébrale (Baker, Wigram \& Gold, 2005; Claeys, Miller, Dalloul-Rampersad, Kollar, 1989; Gilberston, 2006; Guétin, Soua, Voiriot, Picot \& Hérisson, 2009; Magee \& Davidson, 2002; Nayak, Wheeler, Shiflett \& Agostinelli, 2000; Särkämö et al., 2008; Seibert, Fee, Basom \& Zimmerman, 2000; Wheeler, Shiflett \& Nayak, 2003).

Nayak, Wheeler, Shiflett \& Agostinelli (2000) supportent en effet l'efficacité de l'intervention musicale sur les émotions et sur les interactions sociales des personnes ayant subi un AVC ou un TCC. Dans cette étude, deux groupes d'intervention ont été formés: (1) un groupe recevant une thérapie par la musique (chant, composition, pratique d'un instrument, improvisation, écoute de musiques), de pair avec la réadaptation standard; (2) un groupe recevant uniquement la réadaptation standard. L'humeur, les interactions sociales et la participation (implication, motivation, coopération) de tous les participants ont été mesurées par le biais de plusieurs questionnaires remplis par le participant lui-même, la famille et le thérapeute. Les résultats de cette étude montrent un effet positif de la thérapie musicale sur les interactions sociales et la participation aux interventions, de même qu'une tendance à montrer une amélioration de l'humeur et une plus grande motivation comparativement au groupe contrôle. Les auteurs suggèrent que les changements favorables émotifs induits par la musique peuvent exercer un effet positif sur le processus de réadaptation.
Une étude récente (Guétin, Soua, Voiriot, Picot \& Hérisson, 2009) a également montré l'efficacité de la thérapie par la musique, à court et à long terme, dans le traitement de l'humeur dépressive et de l'anxiété chez une population ayant subi un TCC. Dans cette étude, un groupe de personnes ayant subi un TCC sévère ont participé à des séances d'intervention musicale, une fois par semaine, pendant 5 mois. Deux techniques d'intervention musicale ont été utilisées: (1) réceptive (i.e., écoute d'extraits musicaux); et (2) active (i.e., chant, écriture de chansons, exécution de mouvements rythmiques, etc.). Les auteurs ont noté une amélioration significative de l'humeur des participants dès la première séance. L'intervention musicale s'est également montrée efficace à long terme, menant à une réduction significative de la dépression et de l'anxiété des participants à partir de la dixième semaine et jusqu'à la fin de l'étude (après 5 mois).

Par ailleurs, Särkämö et al. (2008) ont montré que l'écoute de musique peut avoir un effet positif sur plusieurs fonctions cognitives et sur l'affectivité des personnes ayant eu un AVC. Dans cette étude, un groupe de participant bénéficiant d'une thérapie par la musique a été comparé à un groupe bénéficiant uniquement de matériel audio non-musical (i.e., histoires sur cassettes) et à un groupe contrôle ne profitant d'aucun matériel auditif. Les auteurs ont noté que les participants ayant bénéficié d'une intervention impliquant l'écoute de musique une à deux heures par jour ont montré une augmentation des capacités d'attention sélective et de mémoire verbale, ainsi qu'une diminution de l'humeur dépressive, comparativement aux participants des deux autres groupes. Ces résultats pourraient par ailleurs se transposer chez d'autres populations cérébrolésées (p.ex., TCC).

L'efficacité de ce type d'intervention est possiblement liée à la préservation de la reconnaissance de certaines catégories d'émotions musicales à la suite d'un TCC. Toutefois, au même titre que pour le visage et la voix, la reconnaissance d'émotions négatives (p.ex., peur) évoquée par des extraits musicaux peut cependant s'avérer déficitaire suivant un TCC, compte tenu des atteintes de certaines structures cérébrales post-accident (p.ex., amygdale). Effectivement, tel que démontré par Gosselin, Peretz, Johnsen et Adolphs (2007) et Gosselin, Peretz et Samson (sous presse), dans le cas de lésions du lobe temporal antéromédian et de l'amygdale, la reconnaissance de la peur évoquée par la musique est déficitaire, et ceci en présence d'une reconnaissance préservée des émotions de joie, d'apaisement et de tristesse évoquées par la musique.

\section{Conclusion}

En résumé, sur la base de la littérature explorée, la reconnaissance de certaines émotions évoquées par les expressions faciales et vocales a été démontrée déficitaire chez les personnes ayant subi un TCC, malgré diverses limites au point de vue méthodologique. Au regard du traitement émotionnel musical suivant un TCC, aucune étude ne s'y est intéressée et pourtant, la musique est utilisée comme intervention en clinique afin de répondre au besoin de prise en charge des patients TCC au plan comportemental et émotionnel. L'efficacité de ce type d'intervention pourrait donc reposer sur la préservation de reconnaissance de certaines émotions musicales après l'accident. Il apparait par ailleurs essentiel d'explorer davantage le sujet puisque la reconnaissance émotionnelle joue un rôle essentiel dans les 
relations interpersonnelles. Ainsi, la reconnaissance d'émotions (surtout faciales et vocales) diminuée postaccident peut mener à des problèmes interpersonnels qui, subséquemment, peuvent contribuer au développement et au maintien de conséquences sur les plans affectif et social (p.ex., dépression, isolement social). Ainsi, le fait d'investiguer davantage la reconnaissance d'émotions évoquées par le visage, la voix et la musique suivant un TCC pourrait éventuellement contribuer au développement d'interventions spécifiques axées sur la reconnaissance des émotions chez les personnes ayant subi un TCC, et par le fait même à optimiser leur participation sociale.

\section{Références}

Adolphs, R. (2002). Recognizing emotion from facial expressions: psychological and neurological mechanisms. Behavioral and cognitive neuroscience reviews, 1, 21-62.

Adolphs, R., \& Damasio, A.R. (2000). Neurobiology of emotion at a systems level. In J.C.E. Borod (Éds.). The neuropsychology of emotion. New York: Oxford University Press.

Adolphs, R., Damasio, H., Tranel, D., Cooper, G., \& Damasio, A.R., (2000). A role for somatosensory cortices in the visual recognition of emotion as revealed by three-dimensional lesion mapping. Journal of neuroscience, 20, 2683-2690.

Adolphs, R., Damasio, H., Tranel, \& Damasio, A.R., (1996). Cortical systems for the recognition of emotion in facial expressions. Journal of neuroscience, 16, 7678-7687.

Adolphs, R., Tranel, D., et al. (1995). Fear and the human amygdala. Journal of Neuroscience, 15(9), 5879-5891.

Adolphs, R., Tranel, D., Damasio, H., \& Damasio, A.R. (1994). Impaired recognition of emotion in facial expressions following bilateral damage to the human amygdala. Nature, 372(6507), 669-672.

Altenmuller, E., Schurmann, K., Lim, V.K., \& Parlitz, D. (2002). Hits to the left, flops to the right: different emotions during listening to music are reflected in cortical lateralization patterns. Neuropsychologia, $40,2242-56$.

Ambadar, Z., Schooler, J., \& Cohn, J. (2005). Deciphering the enigmatic face: the importance of facial dynamics in interpreting subtle facial expressions. Psychological Science, 16, 403-410.

Anderson, C.V., Bigler, E.D., \& Blatter, D.D. (1995). Frontal lobe lesions diffuse damage, and neuropsychological functioning in traumatic brain-injured patients. Journal of Clinical and Experimental Neuropsychology, 17, 900-908.

Arciniegas, D.B., Topkoff, J.L., Rojas, D.C., Sheeder, J., Teale, P., Young, D.A., Sandberg, E., Reite, M.L., \& Adler, L.E. (2001). Reduced hippocampal volume in association with p50 nonsuppression following traumatic brain injury. The Journal of Neuropsychiatry and Clinical Neurosciences, 13, 213-221.

Baker, F., \& Tamplin, J. (2006). Music Therapy Methods in Neurorehabilitation: A Clinician's Manual. London and Philadelphia: Jessica Kingsley Publications.

Baker, F., Wigram, T., \& Gold, C. (2005). The effects of a song-singing programme on the affective speaking intonation of people with traumatic brain injury. Brain Injury, 19(7), 519-528

Baumgartner, T., Lutz, K., Schmidt, C. F., \& Jancke, L. (2006). The emotional power of music: How music enhances the feeling of affective pictures. Brain Research, 1075, 151-64.

Beauchamp, M.H., Ditchfield, M., Catroppa, C., Kean, M., Godfrey, C., Rosenfeld, J.V., \& Anderson, V. (2011). Focal thinning of the posterior corpus callosum: normal variant or post-traumatic? Brain Injury, 25(10), 950-7.

Best, C., Womer, J., \& Queen, H. (1994). Hemispheric asymmetries in adult's perception of infant emotional expressions. Journal of Experimental Psychology: Human Perception and Performance, 20,751-65.

Bibby, H., \& McDonald, S. (2005). Theory of mind after traumatic brain injury. Neuropsychologia, 43, 99-114.

Bigler, E.D. (1999). Neuroimaging in pediatric traumatic head injury: diagnostic considerations and relationships to neurobehavioral outcome. Journal of Clinical Neuroscience, 14, 70-87.

Bigler, E.D., Paver, S., Cullum, C.M., Turkheimer, E., Hubler, D., \& Yeo, R. (1984). Ventricular enlargement, cortical atrophy and neuropsychological performance following head injury. International Journal of Neuroscience, 24, 295-298.

Revista Neuropsicologia Latinoamericana (2011), 3(3), 25-33
Bigler, E.D., Kurth, S., Blatter, D., \& Abildskov, T.J. (1992). Degenerative changes in traumatic brain injury: post-injury magnetic resonance identified ventricular expansion compared to pre-injury levels. Brain Research Bulletin, 28, 651-653.

Bigler, E.D., Johnson, S.C., Anderson, C.V., Blatter, D.D., Gale, S.D., Russo, A.A., Ryser, D.K., Macnamara, S.E., Bailey, B.J., Hopkins, R.O., \& Abildskov, T.J. (1996). Traumatic brain injury and memory: the role of hippocampal atrophy. Neuropsychology, 10, 333-342.

Bigler, E.D., Anderson, C.V., \& Blatter, D.D. (2002). Temporal lobe morphology in normal aging and traumatic brain injury. American Journal of Neuroradiology, 23, 255-266.

Blair, R.J., Morris, J.S., Frith, C.C., Perrett, D.I., \& Dolan, R.J. (1999). Dissociable neural responses to facial expressions of sadness and anger. Brain, 122, 883-893.

Blatter, D.D., Bigler, E.D., Gale, S.D., Johnson, S.C., Anderson, C.V., Burnett, B.M., Ryser, D., Macnamara, S.E., \& Bailey, B.J. (1997). MR-based brain and cerebrospinal fluid measurement after traumatic brain injury: correlation with neuropsychological outcome. American Journal of Neuroradiology, 18, 1-10.

Blood, A.J., \& Zatorre, R.J. (2001). Intensely pleasurable responses to music correlate with activity in brain regions implicated in reward and emotion. Proceedings of the National Academy of Sciences, 98, $11818-11823$.

Blood, A.J., Zatorre, R.J., Bermudez, P., \& Evans, A.C. (1999). Emotional responses to pleasant and unpleasant music correlate with activity in paralimbic brain. Nature Neuroscience, 2(4), 382-387.

Bowers, D., Bauer, R., \& Heilman, K. (1993). The non-verbal affect lexicon: theoretical perspectives from neuropsychological studies of affect perception. Neuropsychology, 7, 433-4.

Bryden, M. P., Ley, R. G., \& Sugarman, J. H. (1982). A left-ear advantage for identifying the emotional quality of tonal sequences. Neuropsychologia, 20, 83-7.

Bunt, L. (2001). Music and emotion: Perspectives from music therapy. In P.N. Juslin, Patrik \& J.A. Sloboda (Eds.). Music and emotion: Theory and research. New York: Oxford University Press.

Cassidy, J.D., Carroll, L., Peloso, P., Borg, J., Von Holst, H., Holm, L., Kraus, J., \& Coronado, V. (2004). Incidence, risk factors and prevention of mild traumatic brain injury: results of the WHO Collaborating Centre Task Force on Mild Traumatic brain Injury. Journal of rehabilitation medicine, 36, 28-60.

Claeys, M.S., Miller, A.C., Dalloul-Rampersad, R., \& Kollar, M. (1989). The role of music and music therapy in the rehabilitation of traumatically brain injured clients. Music Therapy Perspectives, 6 , 71-77.

Cohadon, F. (1992). Biomécanique et physiopathologie des lésions traumatiques crâniennes. In Société de neurochirurgie de langue française, Séminaire d'enseignement, Association des universités partiellement ou entièrement de langue française, UREF (Éds). Traumatismes du crâne et du rachis. Paris : ESTEM.

Critchley, H., Daly, E., Phillips, M., Brammer, M., Bullmore, E., Williams, S., Van Amelsvoort, T., Roberston, D., David, A., \& Murphy, D. (2006). Explicit and implicit neural mechanisms for processing of social information from facial expressions: A functional magnetic resonance imaging study. Human brain mapping, 9, 93-105.

Croker, V. \& McDonald, S. (2005). Recognition of emotion facial expression following traumatic brain injury. Brain Injury, 19 (10), 787-799.

Cullum, C.M., \& Bigler, E.D. (1986). Ventricule size, cortical atrophy and the relationship with neuropsychologicaal status in closed head injury: a quantitative analysis. Journal of Clinical and Experimental Neuropsychology, 8, 437-452.

Dahlberg, C., Hawley, L., Morey, C., Newman, J., Cusick, C.P., \& HarrisonFelix, C. (2006). Social communication skills in persons with post-acute traumatic brain injury: Three perspectives. Brain Injury, 20(4), 425-435.

Damasio, A. (1994). Descartes's error: Emotion, reason and the human brain. London: Papermac.

Davidson, R.J. (1992). Emotion and affective style: hemispheric substrates. Psychological Science, 3, 39-43.

DeGuise E., LeBlanc, J., Feyz, M., Meyer, K., Duplantie, J., Thomas, H., Abouassaly M., Champoux, M-C., Couturier, C., Lin, H., Lu, L., Robinson, C., \& Roger, E. (2008). Long-Term outcome after severe traumatic brain Injury: The McGill interdisciplinary prospective study. The Journal of Head Trauma Rehabilitation, 23 (5), 294-303.

Dimoska, A., McDonald, S., Pell, M.C., Tate, R.L. \& James, C.M. (2010). Recognizing vocal expressions of emotion in patients with social 
skills deficits following traumatic brain injury. Journal of the International Neuropsychological Society, 1-14.

Dolan, R. J., Morris, J. S., \& de Gelder, B. (2001). Crossmodal binding of fear in voice and face. Proceeding of the National Academy of Sciences, 98, 10006-10.

Eldar, E., Ganor, O., Admon, R., Bleich, A., \& Hendler, T. (2007). Feeling the real world: Limbic response to music depends on related content. Cerebral Cortex, 17, 2828-40.

Fecteau, S., Belin, P., Joanette, Y., \& Armony, J. L. (2007). Amygdala responses to nonlinguistic emotional vocalizations. Neuroimage, $36,480-7$.

Flores-Gutierrez, E. O., Diaz, J. L., Barrios, F. A., Favila-Humara, R., Guevara, M. A., del Rio-Portilla, Y., et al (2007). Metabolic and electric brain patterns during pleasant and unpleasant emotions induced by music masterpieces. International Journal of Psychophysiology, 65, 69-84.

Gagnon, L., \& Peretz, I. (2000). Laterality effects in processing tonal and atonal melodies with affective and nonaffective task instructions. Brain \& Cognition, 43, 206-210.

Gale, S.D., Burr, R.B., Bigler, E.D., \& Blatter, D. (1993). Fornix degeneration and memory in traumatic brain injury. Brain Research Bulletin, 32, 345-349.

Gale, S.D., Johnson, S.J., Bigler, E.D., et al. (1995). Trauma induced temporal horn dilatation: neuropsychologic correlates. The Journal of the International Neuropsychological Society, 1, 369370.

George, M.S., Parekh, P.I., Rosinsky, N., Ketter, T.A., Kimberll, T.A., Heilman, K.M., Hercovitch, P. \& Post, R.M. (1996). Understanding emotional prosody activates right hemisphere regions. Archives of neurology, 53, 665-670

Gilberston, S. (2006). Music therapy in early neurorehabilitation with people who have experienced traumatic brain injury. Music Therapy Today, 7(3), 662-693.

Gosling, J. \& Oddy, M. (1999). Rearranged marriages: Marital relationships after head injury. Brain injury, 13, 785-796.

Gosselin, N., Peretz, I., Noulhiane, M., Hasboun, D., Beckett, C., Baulac, M., et al (2005). Impaired recognition of scary music following unilateral temporal lobe excision. Brain, 128, 628-40.

Gosselin, N., Peretz, I., Johnsen, E., \& Adolphs, R. (2007). Amygdala damage impairs emotion recognition from music. Neuropsychologia, 45, 236-244.

Gosselin, N., Peretz, I., \& Samson, S. (sous presse). Impaired recognition of musical emotions and facial expressions following anteromedial temporal lobe excision. Cortex.

Grandjean, D., Sander, D., Pourtois, G., Schwartz, S., Seghier, M.L., Scherer, K.R., \& Vuilleumier, P. (2005). The voices of wrath: Brain responses to angry prosody in meaningless speech. Nature Neuroscience, 8, 145-146.

Green, R.E.A., Turner, G.R., \& Thompson, W.F. (2004). Deficit in facial perception in adults with recent traumatic brain injury. Neuropsychologia, 42, 133-141.

Green, A.C., Baerentsen, K., Stodkilde-Jorgensen, H., Wallentin, M., Roepstorff, A., \& Vuust, P. (2008). Music in minor activates limbic structure: a relationship with dissonance? NeuroReport, 19, 711-715.

Gronwall, D. (1987). Advances in the assessment of attention and information processing after head injury. In H.S. Levin, J. Grafman \& H.M. Eisenberg (Eds.). Neurobehavioral Recovery from Head Injury. New York: Oxford University Press.

Guétin, S., Soua, B., Voiriot, G., Picot, M.C., \& Hérisson, C. (2009). The effect of music therapy on mood and anxiety-depression: an observational study in institutionalised patients with traumatic brain injury. Annals of Physical and Rehabilitation Medicine, 52, 30-40.

Hammond, F.M., Hart, T., Bushnik, T., Corrigan, J.D., \& Sasser, H. (2004). Change and predictors of change in communication, cognition and social function between 1 and 5 years after traumatic brain injury. Journal of head trauma rehabilitation, 19, 324-328.

Haxby, J.V., Hoffman, E.A., \& Gobbini, M.I. (2002). Human neural systems for face recognition and social communication. Biological Psychichiatry, 51, 59-67.

Henry, L.C., Tremblay, S., Boulanger, Y., Ellemberg, D., \& Lassonde, M. (2010) Neurometabolic changes in the acute phase after sports concussions correlate with symptom severity. Journal of Neurotrauma, 27, 65-76.

Hibbard, M.R., Uysal, S., Sliwinski, M., \& Gordon, W.A. (1998). Undiagnosed health issues in individuals with traumatic brain injury living in the community. Journal of Head Trauma Rehabilitation, 13, 47-57.

Hopkins, M.J., Dywan, J., \& Segalowitz, S.J. (2002). Altered electrodermal response to facial expression after closed head injury. Brain Injury, 16 (3), 245-257.

Hornack, J., Bramham, J., Rolls, E., Morris, R., O’Doherty, J., Bullock, P. \& Polkey, C. (2003). Changes in emotion after circumscribed surgical lesions of the orbitofrontal and cingulated cortices. Brain: A journal of neurology, 126, 1691-1712.

Hornack, J., O'Doherty, J., Bramham, J., Rolls, E., Morris, R., Bullock, P. \& Polkey, C. (2004). Reward-related reversal learning after surgical excisions in orbito-frontal or dorsolateral prefrontal cortex in humans. Journal of cognitive neuroscience, 16, 463-478.

Hornack, J., Rolls, E., \& Wade, D. (1996). Face and voice expression identification in patients with emotional and behavioural changes following ventral frontal lobe damage. Neuropsychologia, 34 247-261.

Huisman, T.A., Schwamm, L.H., Schaefer, P.W., Koroshetz, W.J. ShettyAlva, N., Ozsunar, Y., Wu, O., \& Sorensen, A.G. (2004). Diffusion tensor imaging as potential biomarker of white matter injury in diffuse axonal injury. American Journal Of Neuroradiology, 25, 370-376.

Institut canadien d'information sur la santé (2007). Le fardeau des maladies, troubles et traumatismes neurologiques au Canada. Ottawa.

Ietswaart, M., Milders, M., Crawford, J.R., Currie, D., \& Scott, C.L. (2008). Longitudinal aspects of emotion recognition in patients with traumatic brain injury. Neuropsychologia, 46, 148-159.

Jackson, H.F., \& Moffat, N.J. (1987). Impaired emotional recognition following severe head-injury. Cortex, 23, 293-300.

Jantzen, K.J., Anderson, B., Steinberg, F.L., \& Kelso, J.A. (2004). A prospective functional MR imaging study of mild traumatic brain injury in college football players. American Journal of Neuroradiology, 25, 738-745.

Johnson, S.C., Pinkston, J.B., Bigler, E.D., \& Blatter, D.D. (1996). Corpus callosum morphology in normal controls and TBI: sex differences, mechanisms of injury, and neuropsychological correlates. Neuropsychology, 10, 408-415.

Johnstone, T., \& Scherer, K.R. (2000). Vocal communication of emotion. In M. Lewis, \& J.M. Haviland-Jones (Eds.), Handbook of emotions: Second edition. New York: The Gilford Press.

Juslin, P.N. (2001). Communicating emotion in music performance: A review and a theoretical framework. In P.N. Juslin, Patrik \& J.A. Sloboda (Eds.). Music and emotion: Theory and research. New York: Oxford University Press.

Keltner, D., \& Ekman, P. (2000). Facial expression of emotion. In M. Lewis, \& J.M. Haviland-Jones (Eds.). Handbook of emotions: Second edition. New York: The Gilford Press.

Kersel, D.A., Marsh, N.V., Havill, J.H., \& Sleigh, J.W. (2001). Psychosocial functioning during the year following severe traumatic brain injury. Brain Injury, 15, 683-696.

Kewman, D.G., Yanus, B., \& Kirsh, N. (1988). Assessment of distractibility in auditory comprehension after traumatic brain injury. Brain Injury, 2, 131-137.

Knox, L., \& Douglas, J. (2009). Long-term ability to interpret facial expression after traumatic brain injury and its relation to social integration. Brain and Cognition, 69, 442-449.

Kubu, C.S., Casey, R.J., Hanson, T.V., Campbell, D., Roberts, R., \& Varney, N.R. (1993). An investigation of emotion recognition in patients with closed-head injury. Journal of Clinical and Experimental Neuropsychology, 15, 59.

Magee, W.L., \& Davidson, J.W. (2002). The effect of music therapy on mood states in neurological patients: a pilot study. Journal of Music Therapy, 39(1), 20-29.

Mayer, A.R., Mannell, M.V., Ling, J., Gasparovic, C., \& Yeo, R.A. (2011). Functional connectivity in mild traumatic brain injury. Human Brain Mapping, 32(11), 1825-35.

McCullagh, S., \& Feinstein, A. (2005). Cognitive changes. In J.M. Silver, T.W. MacAllister \& S.C. Yudofsky (Eds.). Textbook of traumatic brain injury. Arlington: American Psychiatric Publishing, Inc.

McDonald, S., \& Flanagan, S. (2004). Social perception deficits after traumatic brain injury: Interaction between emotion recognition, mentalizing ability, and social communication. Neurpsychology, 18 (3), 572-579.

McDonald, S., \& Saunders, J.C. (2005). Differential impairment in recognition of emotion across different media in people with severe traumatic brain injury. Journal of the International Neuropsychological Society, 11, 392-399. 
McGowan, J.C., Yang, J.H., Plotkin, R.C., Grossman, R.I., Umile, E.M., Cecil, K.M., \& Bagley, L.J. (2000). Magnetization transfer imaging in the detection of injury associated with mild head trauma. American Journal of Neuroradiology, 21, 875-880.

Menon, V., \& Levitin, D.J. (2005). The rewards of music listening: response and physiological connextivity of the mesolimbic system. Neuroimage, 28, 175-184.

Meythaler, J.M., Peduzzi, J.D., Eleftheriou, E., \& Novack, T.A. (2001). Current concepts: diffuse axonal injury-associated traumatic brain injury. Archives of Physical Medicine and Rehabilitation, 82, 1461-1471.

Milders, M., Ietswaart, M., Crawford, J.R., \& Currie, D. (2008). Scial behacior following traumatic brain injury and its association with emotion recognition, understanding of intentions, and cognitive flexibility. Journal of the International Neuropsychological Society, 14, 318-326.

Mitchell, R.L., Elliott, R., Barry, M. Cruttenden, A. \& Woodruff, P.W. (2003). The neural response to emotional prosody, as revealed by functional magnetic resonance imaging. Neuropsychologia, 41, 1410-1421.

Mitterschiffthaler, M.T., Fu, C.H.Y., Dalton, J.A., Andrew, C.M., \& Williams, S.C.R. (2007). A functional MRI study of happy and sad affective states induced by classical music. Human Brain Mapping, 28, 1150-1162.

Morris, J.S., Frith, C.D., Perrett, D.I., Rowland, D., Young, A.W., Calder, A.J., et al (1996). A differential neural response in the human amygdala to fearful and happy facial expressions. Nature, 383, 812-15.

Morris, J.S., Scott S.K., \& Dolan R.J. (1999). Saying it with feeling: neural responses to emotional vocalizations. Neuropsychologia, 37 (10), 1155-1163.

Morton, M.V., \& Wehman, P. (1995). Psychosocial and emotional sequelae of individuals with traumatic brain injury: A literature review and recommendations. Brain injury, 9, 81-92.

Nayak, S., Wheeler, B.L., Shiflett, S.C., \& Agostinelli, S. (2000). Effect of music therapy on mood and social interaction among individuals with acute traumatic brain injury and stroke. Rehabilitation Psychology, 45(3), 274-283.

Pell, M.D. (2006). Cerebral mechanisms for understanding emotional prosody in speech. Brain and language, 96, 221-234.

Peretz, I. (2001) Listen to the brain: The biological perspective on musical emotions. In J.A. Sloboda \& P. Juslin (Eds.). Music and Emotion: Theory and Research. Oxford: Oxford University Press.

Peretz, I. (2009). Towards a neurobiology of musical emotions. In P. Juslin \& J. Sloboda (Eds.). Handbook of Music and Emotion: Theory, research, applications. Oxford: Oxford University Press.

Picton, T.W., Bentin, S., Berg, P., Donchin, E., Hillyard, S.A., Johnson, R., JR., Miller, G.A., Ritter, W., Ruchkin, D.S., Rugg, M.D. \& Taylor, M.J. (2000). Guidelines for using event-related potentials to study cognition: Recording standards and publication criteria, Psychophysiology, 37, 127-152.

Phillips, M.L., Drevets, W.C., Rauch, S.L., \& Lane, R. (2003). Neurobiology of emotion perception I: The neural basis of normal emotion perception. British Journal of Psychiatry, 54, 504-514.

Phillips, M. L., Young, A. W., Scott, S. K., Calder, A. J., Andrew, C., Giampietro, V., et al (1998). Neural responses to facial and vocal expressions of fear and disgust. Proceedings of the Royal Society of London Series B-Biological Sciences, 265, 1809-17.

Pourtois, G., de Gelder, B., Bol, A., \& Crommelink, M. (2005). Perception of facial expressions and voices and of their combination in the human brain. Cortex, 41, 49-59.

Povlishock, J.T., \& Katz, D.I. (2005). Update of neuropathology and neurological recovery after traumatic brain injury. Journal of Head Trauma Rehabilitation, 20, 76-94.

Regroupement des associations de personnes traumatisées cranio-cérébrales du Québec (2005). Quelques données épidémiologiques d'intérêt quant aux causes du traumatisme cranio-cérébral. [En ligne]. $\begin{array}{lllll}\text { Consulté } & \text { le } & 10 & \text { juillet } & 2009 .\end{array}$ http://www.raptccq.com/raptccq.asp?no=29162

Robinson, R.G., \& Jorge, R.E. (2005). Mood disorders. In J.M. Silver, T.W. MacAllister \& S.C. Yudofsky (Eds.). Textbook of traumatic brain injury. Arlington: American Psychiatric Publishing, Inc.
Särkämö, T., Tervaniemi, M., Laitinen, S., Forsblom, A., Soinila, S. Mikkonen, M., Autti, T., Silvennoinen, H.D., Erkkilä, J., Laine, M., Peretz, I., \& Hietanen, M. (2008). Music listening enhances cognitive recovery and mood after middle cerebral artery stroke. Brain, 131, 866-876.

Schirmer, A., \& Kotz, S.A. (2006). Beyond the right hemisphere: brain mechanisms mediating vocal emotional processing. Trends in Cognitive Sciences, 10(1), 24-30.

Schmidt, L. A., \& Trainor, L. J. (2001). Frontal brain electrical activity (EEG) distinguishes valence and intensity of musical emotions. Cognition \& Emotion, 15, 487-500.

Schmitter-Edgecombe, M., \& Kibby, M.K. (1998). Visual selective selective attention after severe closed head injury. Journal of the International Neuropsychological Society, 4, 144-159.

Scott, S. K., Young, A. W., Calder, A. J., Hellawell, D. J., Aggleton, J. P., \& Johnson, M. (1997). Impaired auditory recognition of fear and anger following bilateral amygdala lesions. Nature, 385(6613), 254-257.

Seibert, P.S., Fee, L., Basom, J., \& Zimmerman, C. (2000). Music and the brain: the impact of music on an oboist's fight recovery. Brain injury, 14(3), 295-302.

Spell, L.A., \& Frank, E. (2000). Recognition of nonverbal communication of affect following traumatic brain injury. Journal of Nonverbal Behavior, 24 (4), 285-300.

Sprengelmeyer, R., Young, A. W., Schroeder, U., Grossenbacher, P. G., Federlein, J., Bütner, T., et al (1999). Knowing to fear. Proceedings in Biological Sciences The Royal Society London B, 266, 2451-6.

Streit, M., Ioannides, A.A., Liu, L., Wolwer, W., Dammers, J., Gross, J., Gaebel, W., \& Muller-Gartner, H.W. (1999). Neurophysiological correlates of the recognition of facial espressions of emotion as revealed by magnetoencephalography. Cognitive brain research, 7 , 481-491.

Tate, D., \& Bigler, E.D. (2000). Fornix and hippocampal atrophy in traumatic brain injury. Learning \& Memory, 7, 442-446.

Thorley, R.R., Wertsch, J.J., \& Klingbeil, G.E. (2001). Acute hypothalamic instability in traumatic brain injury: a case report. Archives of Physical Medicine and Rehabilitation, 82, 246-249.

Tsang, C. D., Trainor, L. J., Santesso, D. L., Tasker, S. L., \& Schmidt, L. A. (2001). Frontal EEG responses as a function of affective musical features. Annals of the New York Academy of Sciences, 930, 439-42.

Umile, E.M., Sandel, M.E., Alavi, A., Terry, C.M., \& Plotkin, P.C. (2002) Dynamic imaging in mild traumatic brain injury: support for the theory of medial temporal vulnerability. Archives of Physical Medicine and Rehabilitation, 83, 1506-1513.

Van Zomeren, A.H., \& Brouwer, W.H. (1994). Clinical Neuropsychology of Attention. New York, Oxford University Press.

Warden, D.L., \& Labbate, L.A. (2005). Posttraumatic stress disorder and other anxiety disorders. In J.M. Silver, T.W. MacAllister \& S.C. Yudofsky (Eds.). Textbook of traumatic brain injury. Arlington: American Psychiatric Publishing, Inc.

Weddell, R.A. (1994). Effetcs of subcortical lesion site on human emotional behaviour. Brain and Cognition, 25, 161-193. Zatorre, R.J., Evans, A.C., Meyer, E. (1994). Neural mechanisms underlying melodic perception and memory for pitch. Journal of neurosciences, 14 , 1908-1919.

Wheeler, B.L., Shiflett, S.C., \& Nayak, S. (2003). Effects of number of sessions and group or individual music therapy on the mood and behavior of people who have had strokes or traumatic brain injuries. Nordic Journal of Music Therapy, 12(2), 139-151.

Yeo, R.A., Gasparovic, C., Merideth, F., Ruhl, D., Doezema, D., \& Mayer, A.R. (2011). A longitudinal proton magnetic resonance spectroscopy study of mild traumatic brain injury. Journal of Neurotrauma, 28(1), 1-11.

Zygun, D.A., Laupland, K.B., Hader, W.J., Kortbeek, J.K., Findlay, C., Doig, C.J., Hameed, S.M. (2005). Severe Traumatic Brain Injury in a Large Canadian Health Region. The Canadian Journal of Neurological Sciences, 32(1), 87-92. 